\title{
All-Optical Signal Processing of Periodic Signals Using a Brillouin Gain Comb
}

\author{
César Jáuregui Misas, Periklis Petropoulos, Member, OSA, and David J. Richardson, Fellow, OSA
}

\begin{abstract}
The amplification of periodic signals using a Brillouin gain comb provides the opportunity to manipulate the amplitude and phase of a signal's individual spectral harmonics and, therefore, its temporal characteristics. In addition to obvious applications in pulse shaping, the approach offers new opportunities in the context of slow-light generation which include power-efficient Brillouin amplification of broadband periodic signals without delay, signal delay/advancement with relatively low gain variation, and fast-light generation accompanied by high levels of gain.
\end{abstract}

Index Terms-All-optical signal processing, Brillouin scattering, fast light, slow light.

\section{INTRODUCTION}

$\mathbf{O}$ VER the years, Brillouin amplification has been used to develop a multitude of devices, including sensors [1], microwave signal-processing systems [2], and phase-to-amplitude-modulation converters [3]. These devices have, in general, only exploited the power gain associated with the Brillouin process. However, there has been a growing appreciation that the phase changes associated with Brillouin amplification can also be of use, in particular, within the context of slow-light generation. Brillouin-assisted slow-light generation, as first demonstrated in optical fibers in 2005 [4], [5], exploits the gain and phase change associated with Brillouin amplification, supporting simultaneous amplification and slowing of light pulses. The approach offers several advantages over traditional slow-light generation techniques. In particular, it is wavelength independent, can be generated at room temperature using off-the-shelf equipment, and it takes place directly within an optical fiber which makes it inherently compatible with today's telecommunication systems.

However, despite its many advantages, Brillouin-assisted slow light has two significant drawbacks: 1) the requirement for high pump powers to obtain significant delays and 2) the narrow bandwidth of the Brillouin gain peak which is insufficient to accommodate high-speed telecommunication signals. The first problem has been addressed by choosing appropriate

Manuscript received November 27, 2007; revised March 12, 2008. Current version published December 19, 2008. The first author was supported by the Fundación Ramón Areces.

C. Jáuregui Misas is with the Optoelectronics Research Centre, University of Southampton, Southampton SO17 1BJ, U.K. and also with the FriedrichSchiller-University Jena, Institute of Applied Physics, Jena 07745, Germany (e-mail: cesar.jauregui@gmail.com).

P. Petropoulos and D. J. Richardson are with the Optoelectronics Research Centre, University of Southampton, Southampton SO17 1BJ, U.K. (e-mail: pp@orc.soton.ac.uk; djr@orc.soton.ac.uk).

Color versions of one or more of the figures in this paper are available online at http://ieeexplore.ieee.org.

Digital Object Identifier 10.1109/JLT.2008.925041 highly nonlinear optical fibers [6], [7]. This approach reduces the pump power demands by about one order of magnitude with respect to conventional silica fibers, with at least one further order of magnitude in prospect using higher nonlinearity materials. However, the second problem associated with the Brillouin gain bandwidth has sparked further developments and research into the Brillouin scattering process itself. In order to extend the Brillouin gain bandwidth to the multi-Gigahertz regime, several groups have broadened the spectrum of the pump by modulating it with a pseudorandom bit sequence (PRBS) [8], Gaussian noise [9], or using phase modulation [10]. This approach highlights the strong dependence of the Brillouin scattering on the characteristics of the pump [11], which thereby allows tailoring of the gain profile. Thus, for example, some groups have mimicked the spectrum of electromagnetic-induced transparency [12], [13], obtaining higher delays than otherwise possible.

In this paper, we exploit the narrowband gain profile of stimulated Brillouin scattering and the use of multiwavelength pumps to create a Brillouin gain comb with which to amplify and process temporally periodic signals (which also have a comb spectrum in the frequency domain). We focus, in particular, on the case that the gain comb has a frequency spacing that is close or equal to the signal frequency, or indeed a higher harmonic of this frequency. In this way, amplitude and/or phase manipulation of the individual signal harmonics can be achieved, providing a very flexible way to reshape and retime periodic signals. We show that this approach allows for phenomena not previously seen with conventional Brillouin-assisted slow light and which includes signal amplification without delay, delay tuning with little gain variation, and fast-light generation with high gain. It is also important to note that, unlike the case of PRBS-based pump spectrum broadening, there is no fundamental frequency limit associated with this technique (i.e., the signal can have a frequency higher than twice the Brillouin shift). Moreover, as long as one can generate a Brillouin gain comb with the appropriate spacing, the technique is independent of the signal repetition rate (both in terms of processing performance and power requirement).

This paper is divided into four sections. The operating principle is presented and discussed in Section II, and some experimental results that illustrate the capabilities of the technique are analyzed in Section III. Finally, conclusions are drawn in Section IV.

\section{OPERATING PRINCIPLE}

From Fourier analysis, any signal that is periodic in the temporal domain exhibits a spectrum comprising discrete spectral 

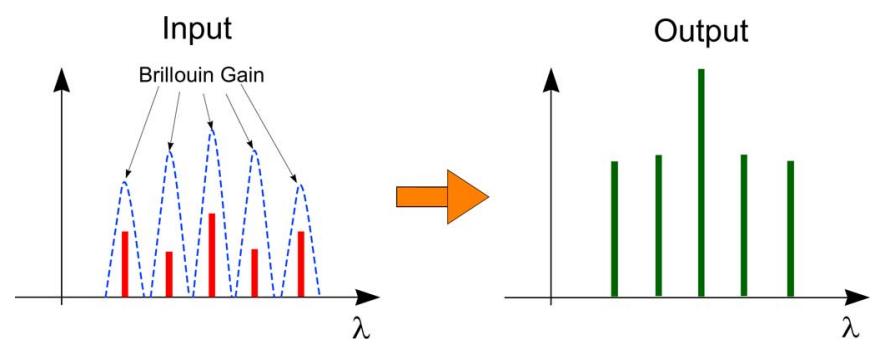

Fig. 1. Operating principle of the proposed signal-processing technique: a Brillouin gain comb is generated such that the gain lines have similar spacing to the signal harmonics. Each Brillouin gain line individually modifies one harmonic of the signal. The combined effect of the Brillouin gain comb gives rise to a modified output spectrum and, therefore, to a reshaped signal. Note that phase as well as the amplitude of each individual harmonic may be altered as a result of the overall amplification process.

harmonics in the frequency domain. These spectral harmonics are each essentially delta functions and are thus very much narrower than the inherent Brillouin linewidth ( $\sim 50 \mathrm{MHz})$. Assuming that the harmonic frequency spacing is much greater than the Brillouin linewidth, it becomes possible to provide gain for a single harmonic component of a periodic signal using a single Brillouin gain peak. The precise gain and phase shift experienced by the electrical field are determined by the amplitude and relative position of the Brillouin gain peak as shown in (1)

$$
\begin{aligned}
g_{B}(\omega) & =\frac{g_{o}}{2} \frac{P_{p}}{A_{\text {eff }}} \frac{L}{1+\left[2 \frac{\left(\omega-\Omega_{B}\right)}{\Gamma_{B}}\right]^{2}}\left[1+2 j \frac{\left(\omega-\Omega_{B}\right)}{\Gamma_{B}}\right] \\
& =\frac{1}{2} G(\omega)+j \phi_{s}(\omega) \cdot L .
\end{aligned}
$$

Here, the signal is considered to travel in the $+\mathrm{z}$ direction, $g_{o}$ is the Brillouin gain coefficient which depends on the material of the optical fiber, $A_{\text {eff }}$ is the effective mode area of the fiber, $P_{p}$ is the pump power, $\omega$ is the angular frequency shift from the pump wavelength $\left(\omega_{p}\right), \Omega_{B}$ is the Brillouin angular frequency shift, $\Gamma_{B}$ is the FWHM bandwidth of the Brillouin gain profile, $L$ is the length of the fiber, and $j$ is the imaginary unit. On the other hand, $G(\omega)$ is the power gain, and $\beta(\omega)=\phi_{s}(\omega) \cdot L$ is the imaginary part of the gain that introduces a change in the propagation constant of the electric field.

The concept can obviously be extended to the case of multiple harmonics by incorporating additional Brillouin gain peaks. Fig. 1 shows an example of how an input spectrum is shaped via the interaction with a Brillouin gain comb-where the gain and phase shift experienced by each harmonic are determined by the properties of the local gain peak according to (1). From a practical perspective, a Brillouin gain comb is most straightforwardly obtained by generating a suitable frequency comb for the pump-each pump line generating an associated gain line. This can be achieved either by using an array of independent lasers (allowing, in principle, any desired gain comb to be created), or more simply, by either external modulation [14] or direct modulation of a single laser as we show herein.

To facilitate discussion of the various effects that one can obtain using Brillouin gain comb amplification, consider the periodic gain spectrum with just three peaks shown in Fig. 2. Several

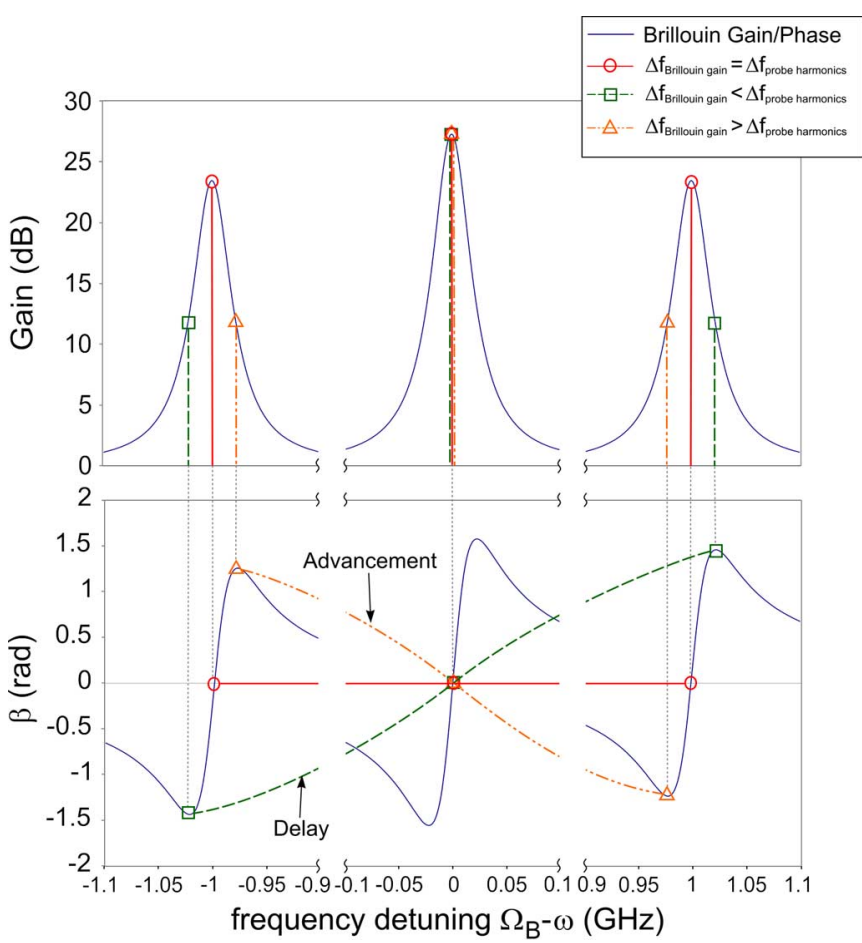

Fig. 2. Result of having slightly different Brillouin gain frequency spacing and probe harmonic separation. The graph shows the Brillouin gain (solid line above) and the Brillouin-induced phase change $\beta$ (below). As can be seen, under these circumstances, each probe harmonic (squares or triangles) undergoes a different phase change; as opposed to the situation with matched gain frequency spacing and probe harmonic separation (circles). This situation is equivalent to having a single broader gain line ( $2 \mathrm{GHz}$ in this example) as illustrated by the dashed and double-dotted lines in the graph below. When the phase change varies monotonically with frequency, it gives rise to either slow (equivalent Brillouin gain imaginary part increasing with frequency) or fast light (equivalent Brillouin gain imaginary part decreasing with frequency). This, in turn, generates delay or advancement in the processed signal, respectively.

interesting situations can arise depending on the respective frequencies of the gain comb and signal, along with their relative alignment.

In the case that the frequency spacing of the Brillouin gain comb $\left(\Delta f_{\text {Brillouin gain }}\right)$ matches the probe harmonic separation $\left(\Delta f_{\text {probe harmonics }}\right)$ all of the probe harmonics will undergo the same amount of phase change due to the Brillouin gain (see the lines with circles in Fig. 2). In particular, if the harmonics are placed at the peaks of the gain, then this phase shift will be zero. If the gain per line is equal for each harmonic, then the periodic signal will be faithfully amplified without distortion despite the fact that the full bandwidth of the signal can greatly exceed the bandwidth of the individual Brillouin gain peaks. Alternatively, if the gain per harmonic is different, then amplitude-based pulse shaping becomes possible.

Other opportunities emerge when $\Delta \mathrm{f}_{\text {Brillouin gain }}$ is different to $\Delta f_{\text {probe harmonics. Under these circumstances, each }}$ harmonic will suffer a different phase change, as depicted in Fig. 2 (lower graph). This automatically means that there will be some amount of dispersion that, when properly managed, can lead to a group velocity change and, hence, to signal delay or advancement. The physical mechanism can be better understood by plotting the amount of gain/phase change for each harmonic (squares, circles, and triangles) against frequency 


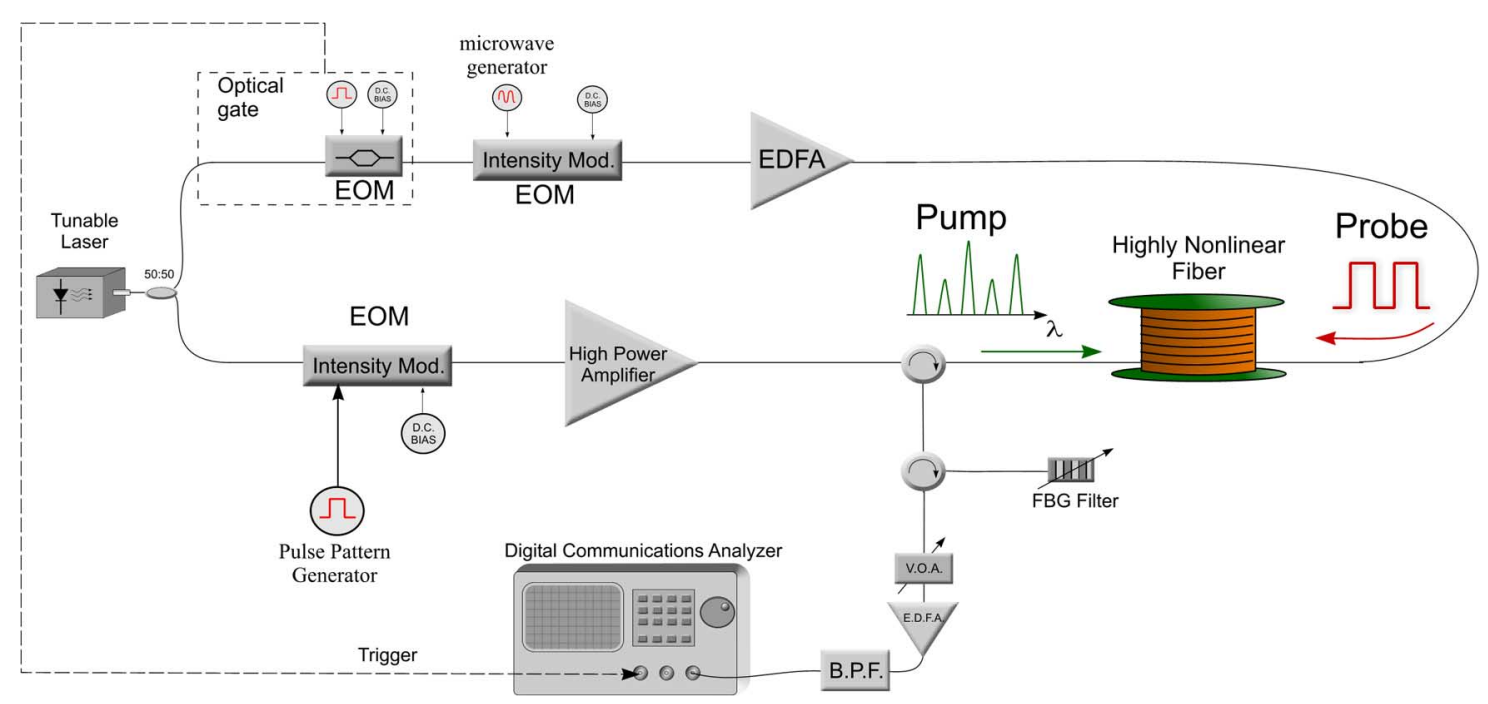

Fig. 3. Experimental setup.

and drawing a line connecting the different points. If all of the gain peaks are equally spaced, and all of the harmonics lie within the linear region of the imaginary part of the Brillouin gain (which corresponds to the rapid transition areas in Fig. 2), then these phase changes vary linearly with optical frequency. This means that the group velocity of the signal will change by a constant value, thus leading to controllable signal delay or advancement. Under these circumstances, the Brillouin comb behaves exactly as a single broadened Brillouin gain line as far as the periodic signal is concerned. It is important to note that although the harmonics are slightly detuned from the gain peak (the maximum phase change occurs at offset frequencies of $\sim \Gamma_{B} / 2$ ), the signal still experiences high gain, as clearly seen in the upper graph in Fig. 2. Thus, in contrast to conventional Brillouin-assisted slow light, it is possible to generate fast light with a significant amount of gain with this technique. Amplitude- and phase-based pulse shaping are also now possible, although it is to be appreciated that these two control parameters are coupled.

From the simple considerations that follow, it is possible to predict the maximum amount of delay/advancement induced in the signal. It is known that the delay is given by

$$
\tau=\frac{d \beta}{d \omega} .
$$

Consequently, using the equivalent model shown in Fig. 2, it is possible to obtain the following equation that predicts the amount of delay/advancement induced by a certain Brillouin gain comb (provided that all of the harmonics lie in the linear part of the phase change) from the slope of the line connecting the Brillouin-induced phase change on each harmonic

$$
\tau=\frac{d \beta}{d \omega} \approx \frac{\Delta \beta}{2 \pi \cdot\left(N_{\text {lines }}-1\right) \cdot f_{m}}
$$

where $\Delta \beta$ is the difference between the imaginary part of the Brillouin gain (parameter $\beta$ as defined in (1)) seen by the highest frequency harmonic and that of the lowest frequency harmonic; $N_{\text {lines }}$ is the number of Brillouin gain lines in the comb; and $f_{m}$ is the frequency separation of the probe harmonics. Note that a positive value in (3) means delay, whereas a negative value implies advancement. As can be seen, the greater the number of gain lines, the lower the total induced delay (because the higher the frequency span covered by the equivalent gain line, the smaller the equivalent dispersion). Unfortunately, this counters the requirement for amplification without distortion that demands the highest possible number of equal gain lines. A compromise must therefore be met.

\section{EXPERIMENTAL RESULTS}

In this section, we will present different experimental results that highlight the flexibility and potential of the proposed technique. The section is divided into two different subsections covering signal reshaping and signal retiming separately.

A simplified diagram of the experimental setup is shown in Fig. 3. As can be seen, both pump and probe are generated in our experiments from a single laser source to avoid the detrimental effects of wavelength drift, note though that this is not a fundamental requirement. However, it is to be appreciated that this way of synthesizing the probe also results in other unwanted components, in particular, the original carrier and another sideband which need to be eliminated. In our setup, these unwanted components are rejected by an FBG filter incorporated within the detection system. This filter has a bandwidth of $\sim 0.2 \mathrm{~nm}$ and we use one of its edges to separate pump and probe. Unfortunately, the filter edge is not as steep as we would ideally like and it also partially filters out the highest frequency signal harmonic, providing an additional degree of signal distortion.

The fiber used to generate the Brillouin gain is a 490-m-long highly nonlinear silica fiber that has a $10.3 \mu \mathrm{m}^{2}$ effective area and a loss of $0.49 \mathrm{~dB} / \mathrm{km}$. This fiber has a Brillouin frequency shift of $\sim 9.3 \mathrm{GHz}$ and a Brillouin gain FWHM bandwidth of $\sim 52 \mathrm{MHz}$.

\section{A. Comb Generation Technique}

In our setup, the pump comb is generated by passing the harmonic content of a periodic electronic modulating signal to the laser light through direct amplitude modulation using an 


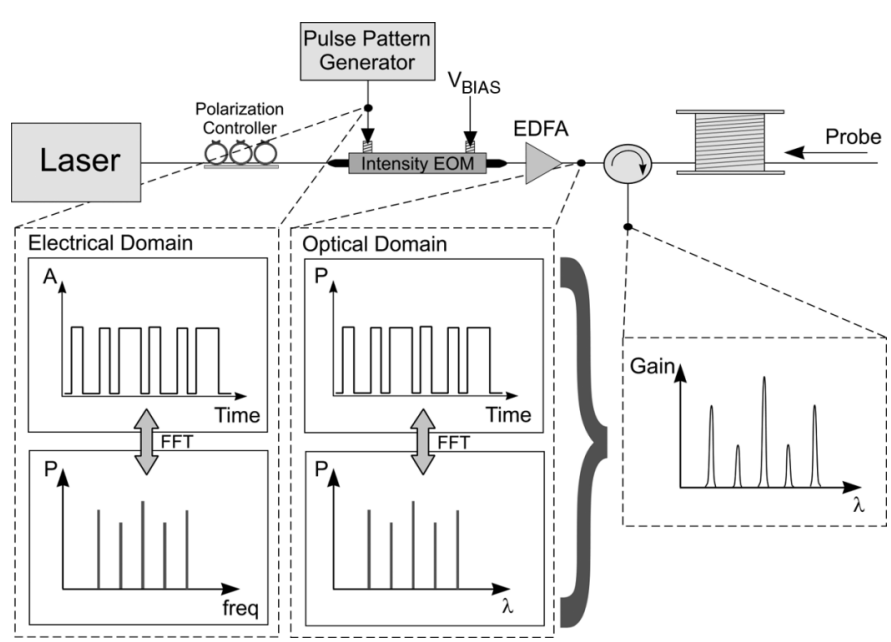

Fig. 4. Schematic of the comb generation setup. The light is amplitude modulated by a pulse-patterned signal to give rise to the desired optical comb. This can subsequently be amplified and used as the pump for Brillouin-assisted signal processing.

electro-optic amplitude modulator, as illustrated in Fig. 4. Contrary to the system in [14], this makes it possible to easily determine the final optical comb structure simply by obtaining the electrical spectrum of the modulating signal. This characteristic represents a considerable advantage of the technique and greatly facilitates the inclusion of an electronic control loop to obtain a predefined comb.

In our particular implementation, as seen in Fig. 4, the modulating signal was a periodic square pulse pattern obtained from a pulse pattern generator (PPG). This provides good control of the optical comb (both in terms of the relative amplitude of the harmonics and in their spacing) through simple changes in the pattern and clock frequency. However, the use of pulse patterns, although flexible, does not allow the synthesis of any arbitrary comb. In order to improve upon this, an arbitrary waveform generator would be required. However, even in this case, the harmonics will always be symmetric around the dc component and will, therefore, only be controllable in pairs. An additional mechanism (more complex modulation, or some additional optical filtering technique) would be required to break the symmetry and allow control of individual harmonics.

Once the pump comb seed is generated, it needs to be amplified to a suitable power level and passed through the HNLF to generate the Brillouin gain comb. This final gain comb is related to the optical comb used as a pump; however, it does not necessarily preserve the relative height of the harmonics due to the exponential relation between pump power and Brillouin gain as is particularly evident at high Brillouin gains.

One issue of concern is the possible amplitude-modulation transfer from the pump to the probe through the Brillouin gain. However, if the fiber is long enough in order to accommodate several periods of the pump, the amplitude variations are averaged out by the counterpropagating nature of the Brillouin interaction. Thus, in our experiments, we did not observe any trace of amplitude modulation in the amplified probe.

This comb generation technique is very simple, inexpensive, and is ideally suited for those applications where an optical
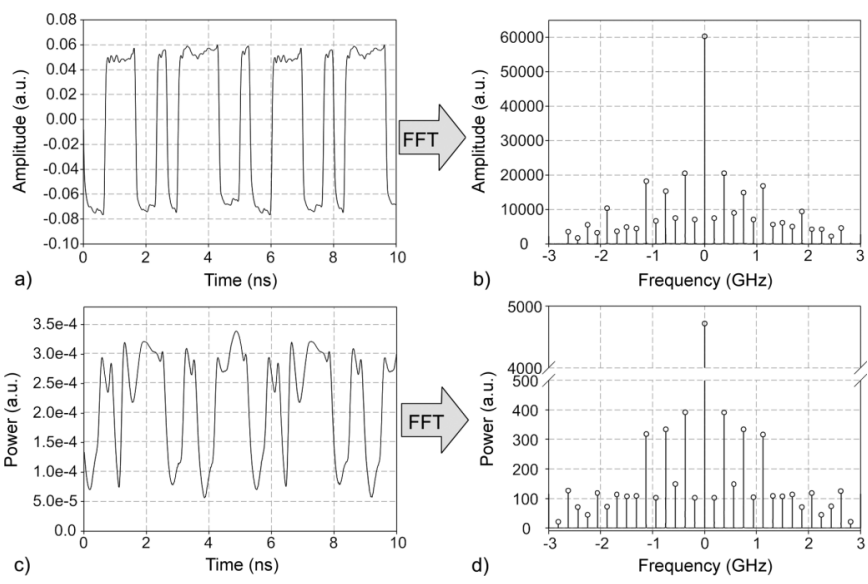

Fig. 5. Experimental results on optical comb generation. The electrical modulating signal (a) exhibits a spectral content appropriate to generate a seven-line comb (b). This signal is used to modulate the light obtaining an optical signal (c) whose spectrum (d) closely resembles that of the electrical signal.

comb with dynamic reconfiguration is required (laboratory, individual WDM channel control, etc). However, the technique presents certain limitations that might reduce its applicability. Its main drawback is that it requires the modulating signal to have spectral components at frequencies higher than the harmonic separation as opposed to the case of cascaded modulators driven with a sine wave [14]. From a practical point of view, this implies that the clock frequency of the pulse pattern generator needs to be much higher than the final harmonic spacing of the optical comb and this becomes progressively more of an issue as higher numbers of harmonics are desired.

For one of the experiments described in the next section, we required a 7-line Brillouin gain comb with almost equal gain lines separated by $375 \mathrm{MHz}$. The required electrical pulse pattern is shown in Fig. 5(a). This periodic pulse train was obtained with a PPG clock frequency of $\sim 3 \mathrm{GHz}$ and a pattern period of $\sim 5 \mathrm{~ns}$. The electric spectrum of the modulating signal is shown in Fig. 5(b). Six harmonics of almost equal amplitude can be seen. Note the relatively high-level dc signal component associated with this data pattern. In principle, this could dominate the Brillouin gain spectrum; however, the magnitude of this component in the optical domain can be reduced through control of the modulator bias, such that the modulator operates close to the minimum transmission point. This results in a degree of pattern distortion as can clearly be seen in Fig. 5(c); however, other than a slight power redistribution between higher harmonics, the optical signal still retains most of the harmonic content of the original electrical signal. Note that the optical spectrum still exhibits a large dc component ( $\sim 10$ times larger than the higher harmonic); however, in this instance, this component arises largely due to amplified spontaneous emission (ASE) from the amplifier used to boost the signal. Nevertheless, although this is seen as an offset level by the photodetector, it does not lead to significant Brillouin gain due to the broadband nature of ASE.

When the pump signal shown in Fig. 5(b) is launched into the 490-m-long highly nonlinear fiber, it generates the Brillouin gain comb seen in Fig. 6. It can be seen that it comprises seven almost equal gain lines separated by $375 \mathrm{MHz}$. Lower gain peaks coming from smaller harmonics in Fig. 5(d) though 


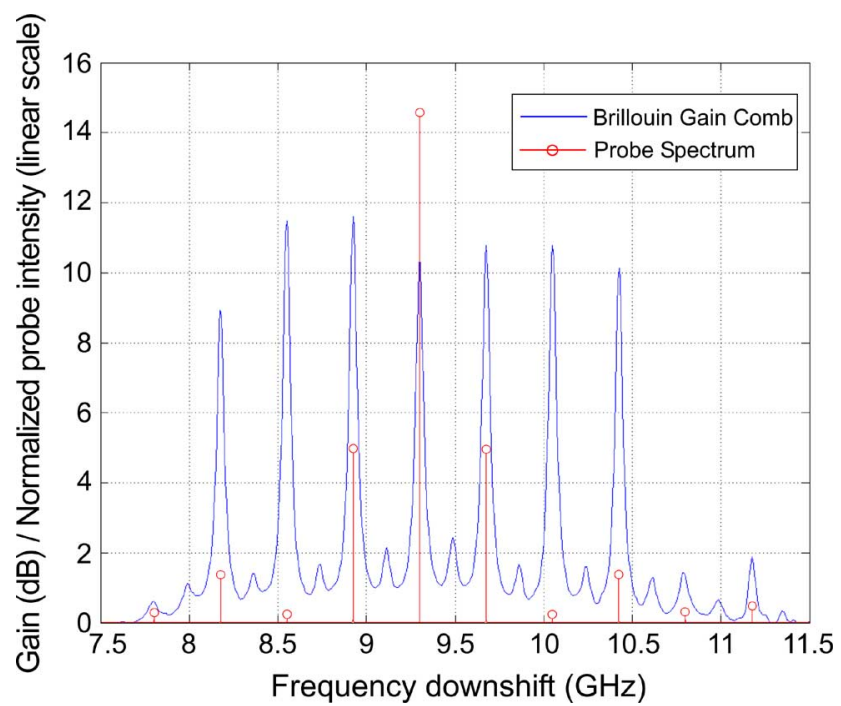

Fig. 6. Experimentally measured Brillouin gain comb (solid line) superimposed upon the Fourier transform of the probe signal (lines topped with dots). Note how the gain lines are placed only around the harmonics of the signal.

can also be seen. It is worth noticing that other than the absence of the large dc component, the Brillouin comb mimics the spectrum of the pump signal as desired.

\section{B. Signal Amplification and Reshaping}

As described previously, in contrast to conventional Brillouin amplification, this optical signal-processing technique can be used for signal amplification and reshaping without incurring any extra delay (provided $\Delta \mathrm{f}_{\text {Brillouin gain }}=\Delta \mathrm{f}_{\text {probe harmonics }}$ ). This is the operating regime used in the experiments that we present in this subsection.

The first experiment that we carried out to highlight the potential of the technique was the amplification of a square pulse train. The repetition rate and duty cycle of the signal pulse train were chosen to be $375 \mathrm{MHz}$ and $50 \%$, respectively. Note that the frequency of the pulse train is almost one order of magnitude higher than the Brillouin gain bandwidth. Unless pump broadening techniques [8]-[10] were used, it would not be possible to amplify this signal by a single Brillouin gain line. However, the use of a spectrally broadened pump is highly inefficient for periodic signals since it provides gain at frequencies where there is no signal content. This introduces a pump power penalty (i.e., the pump power required to keep constant the relative delay or gain scales linearly with signal bandwidth-by relative delay, we mean the ratio of the delay to signal period) and much higher noise in the amplified signal. Instead, as illustrated in Fig. 6, our technique places the gain exactly where needed (i.e., around the harmonics of the signal). This feature makes this technique far more pump efficient and less noisy. It is also to be appreciated that the power requirements are independent of repetition frequency since processing a faster input signal only requires a replacement of the gain peaks around the new harmonics - the number of harmonics remains the same. This characteristic clearly differentiates the proposed technique from the conventional Brillouin gain-broadening approach for which there is a strong pump power penalty associated with the use of broader gain bandwidths.

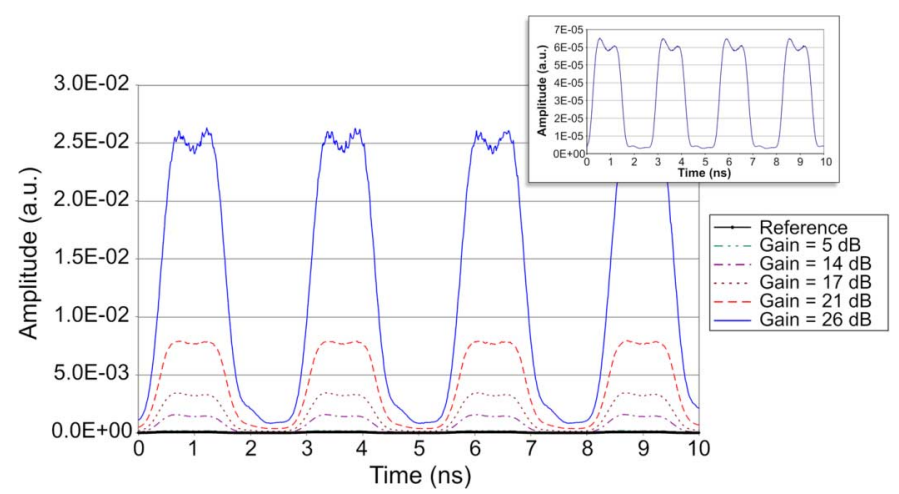

Fig. 7. Square pulse train amplification using a seven-line Brillouin comb of approximately equal gain lines. High gain values can be achieved $(\sim 26 \mathrm{~dB})$ with relatively little distortion. Note that there is no delay induced in the amplified signals. The inset shows the input signal.

Fig. 6 gives a clear insight into the flexibility of the technique. Not only is it possible to change the Brillouin gain comb (solid line) at will, but one can also choose to center it at different harmonics of the probe (lines topped with dots). Each reconfiguration of the comb will generate a different output waveform since each modifies the spectral content of the signal in a different way.

As seen before, the Brillouin comb of Fig. 6 comprises seven almost equal amplitude gain lines spaced by $375 \mathrm{MHz}$. The first (highest optical frequency) gain line appears to be smaller than the rest because it has been partially filtered out by the FBG filter used to reject the pump and unwanted signal components at the receiver. It is important to point out at this point that the partial filtering of the first gain line accounts for some of the distortion observed in Fig. 6. Thus, even better results can be expected if a sharper filter (or an alternative experimental configuration) is used. Thus, if we apply this Brillouin gain comb to any periodic signal whose energy is concentrated in its first seven harmonics, we can expect to obtain amplification with little distortion. This is demonstrated in Fig. 7 in which the output signals are presented for increasing Brillouin gain. Comparing these output signals with the input probe presented in the inset, it can be seen that the technique offers up to $\sim 26-\mathrm{dB}$ gain with relatively low distortion.

Another feature of Fig. 7 worth highlighting is the absence of any induced delay in the amplified waveforms. This result confirms the theoretical predictions and hints toward the decoupling between gain and delay offered by this technique.

In order to demonstrate the frequency-independent power requirement feature of this technique, we increased the frequency of the input square pulse train to $1.06 \mathrm{GHz}$ and again generated a Brillouin comb with the appropriate gain-line spacing. By progressively increasing the pump power to roughly the same maximum value used in Fig. 7, we obtained the results presented in Fig. 8. This graph shows a maximum gain of $\sim 28 \mathrm{~dB}$ and clearly demonstrates that this technique does not suffer any kind of pump power penalty with input signals of a broader bandwidth. These results exhibit higher distortion than in the 375-MHz case, which we attribute to the more severe filtering of the first two gain peaks in this case by the FBG filter (these peaks are pushed out closer toward the pump at the 


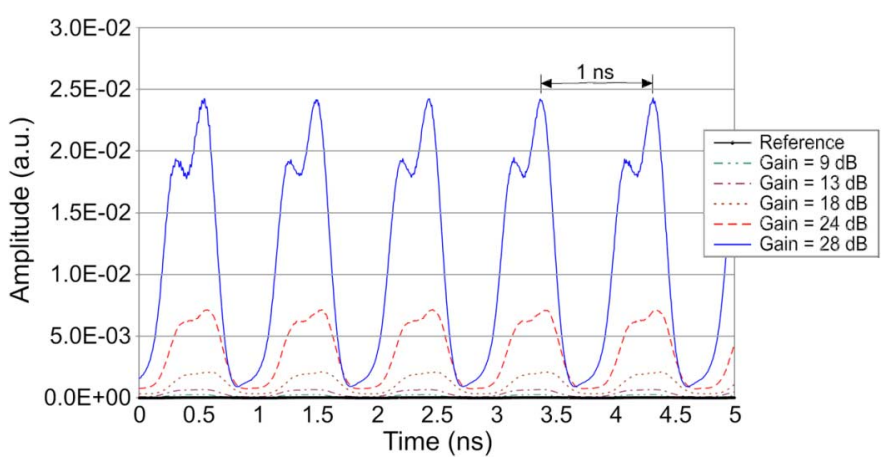

Fig. 8. Amplification of a $1.06-\mathrm{GHz}$ square pulse train. A gain of $\sim 28$ $\mathrm{dB}$ has been obtained, which highlights that there is no pump power penalty at higher frequencies.

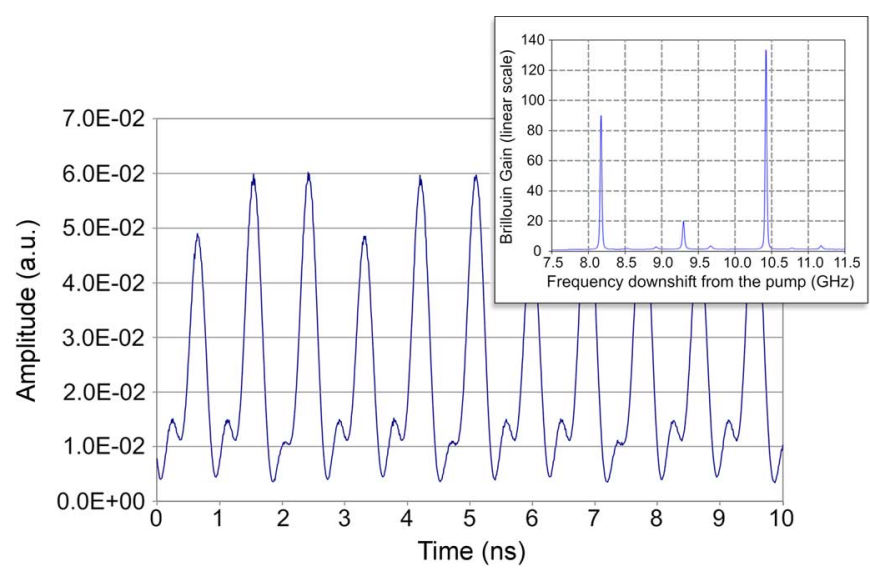

Fig. 9. Result of $3 \times$ frequency multiplication reshaping. The input is a $375-\mathrm{MHz}$ square pulse train whereas the output exhibits $1.125-\mathrm{GHz}$ frequency. The inset shows the Brillouin gain comb used in this experiment.

higher frequency so the filter is even less optimal). This also explains the small timing advancement observed as the probe is amplified.

Finally, in order to demonstrate the versatility of the technique, we changed the Brillouin gain comb to a three-line spectrum with $1.125-\mathrm{GHz}$ gain spacing in an attempt to reshape the input signal. This frequency spacing coincides with the third harmonic of the $375-\mathrm{MHz}$ square train pulse. This comb can be seen in the inset of Fig. 9. When applying this Brillouin gain comb to the square train, a quasisinusoidal signal emerges at the output with a repetition rate which is three times higher than that of the input, as shown in Fig. 9. The Brillouin gain was $\sim 28 \mathrm{~dB}$ in this instance. Note that the different peaks are not equal because the amplified harmonics are not sufficiently strong relative to the rest of the harmonic content of the signal (i.e., that of the input probe) to render its impact negligible. Higher pump power would have been required to equalize all of the pulses.

In conclusion, our results show that the technique provides simultaneous signal reshaping and amplification without retiming, and highlights the relative power efficiency of the approach for Brillouin amplification of broadband periodic signals.

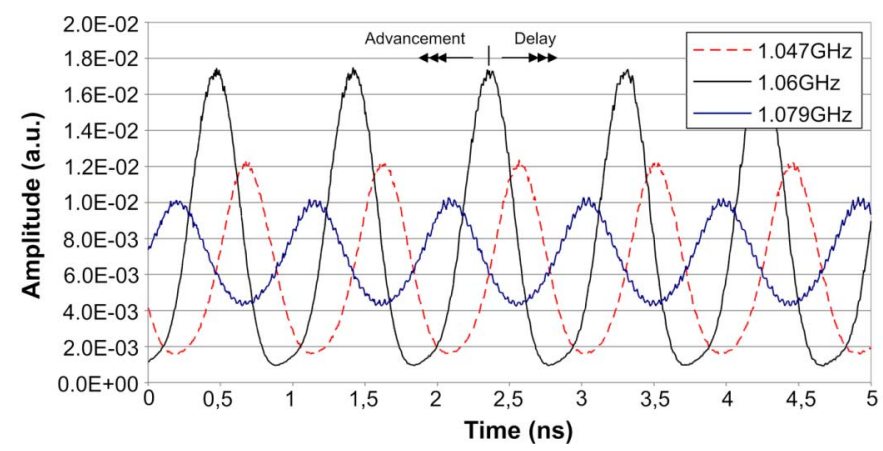

Fig. 10. Experimental results for slow/fast-light generation. The delay/advancement is generated by using Brillouin gain combs with slightly different gain spacing. The signals with maximum delay and advancement (solid lines) have comparable amplitudes which demonstrate the generation of fast light with high gain.

\section{Signal Retiming}

As highlighted by (3), the best situation in order to obtain the largest delays/advancements and, therefore, experimentally demonstrate the generation of slow/fast light with a Brillouin gain comb (at any given probe frequency) is to work with the minimum number of gain lines. In this case, we chose to apply three gain lines to a 1.06-GHz square pulse train. Under these circumstances, the output signal will be heavily reshaped (it should have a quasisinusoidal shape). However, in this section, it is primarily just the delay/advancement that we want to study.

The graph shown in Fig. 2 actually corresponds to the simulation of the three-gain line Brillouin comb used in the experiments for the highest pump power configuration. This means that this graph can be used to estimate the maximum delay and advancement expected in the experiments. Thus, introducing the appropriate $\Delta \beta$ (obtained from Fig. 2) in (3) and considering just three gain lines, a maximum delay of $\sim 238$ ps and a maximum advancement of $\sim-200 \mathrm{ps}$ are obtained. Note that the expected advancement is smaller than the delay. This is because the imaginary part of the Brillouin gain for each harmonic actually extends to the next gain peak (see Fig. 2) thus generating a nonzero phase contribution within it. This contribution is positive for positive frequency detunings from the Brillouin frequency shift, and negative for negative detunings. Thus, the harmonic at the righthand side of Fig. 2 will present a small positive phase offset, whereas the lefthand one will have a small negative offset. This way, the $\Delta \beta$ for pulse advancement will be smaller than the corresponding one for delay.

Using a Brillouin gain of about $26 \mathrm{~dB}$ and progressively changing the Brillouin gain line spacing from $1.047 \mathrm{GHz}$ up to $1.079 \mathrm{GHz}$, we obtained the results shown in Fig. 10. Here, the delay/advancement of the output signal can clearly be seen. Note that the signal can be shifted by almost an entire semiperiod ( $\sim 500 \mathrm{ps})$, with relatively little change in gain. This is an improvement over normal slow-light systems in which this amount of delay change would be accompanied by a strong gain variation $(\sim 30 \mathrm{~dB})$.

Another unique feature of this technique is that it is able to generate fast light with the same amount of gain as slow light. Furthermore, generating signal advancement here is very simple: it only requires a change of the Brillouin gain line 


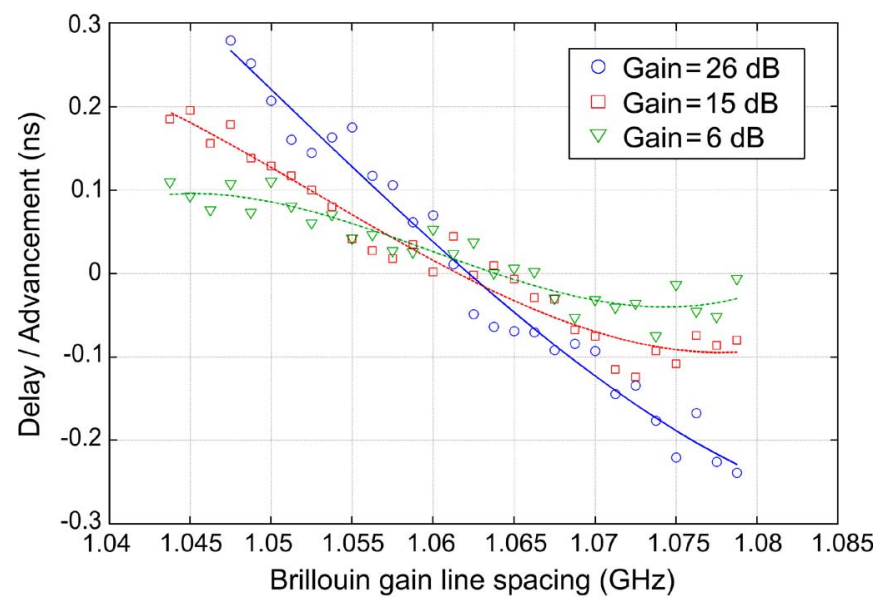

Fig. 11. Experimental measurement of delay (positive value) or advancement (negative values) for three Brillouin gains: $26 \mathrm{~dB}$ (circles), $15 \mathrm{~dB}$ (squares), and $6 \mathrm{~dB}$ (triangles). The solid lines represent the theoretical prediction for the delay/advancement.

spacing to ensure that it is higher than the probe harmonic separation (the opposite situation of that needed to generate delay). Thus, this system of control offers a smooth and continuous transition between slow and fast-light regimes (in contrast to traditional Brillouin-assisted slow-light techniques).

In order to understand the results more readily, the delay/advancements shown in Fig. 10 are plotted against the Brillouin gain line spacing in Fig. 11 (circles). This process is repeated for another two Brillouin gains: $15 \mathrm{~dB}$ (squares) and $6 \mathrm{~dB}$ (triangles). All three lines in Fig. 11 exhibit quasilinear behavior for small frequency detuning between pump and signal, but it saturates for larger detuning values. This is due to the fact that when the Brillouin gain line spacing is very different to probe harmonic separation, the harmonics fall out of the region of linear phase change shown in Fig. 2. This means that the harmonics go beyond the frequencies corresponding to the peaks of maximum and minimum Brillouin-induced phase change and, therefore, the overall $\Delta \beta$ saturates at first (while the harmonics are around the peaks) and then decreases beyond this. This behavior establishes the limits for the maximum achievable delay/ advancement. These maximum values are exactly the same as those that would be theoretically obtained in a conventional pump-broadened slow-light system [8]-[10] of equivalent gain and bandwidth. Moreover, as in conventional Brillouin-assisted slow-light schemes, the maximum achievable delays/advancements are a direct function of the injected pump power as seen in (1).

It can be seen that the values for the maximum delay and advancement in Fig. 11 agree reasonably well with the simple calculations done previously, and with the theoretical prediction obtained by our simulations (solid line). It is also worth noting that as predicted, the amount of delay is higher than the amount of signal advancement for all pump powers.

As expected, the higher the Brillouin gain, the higher the achievable delay/advancement. However, since the gain has a strong impact on the output waveform shape, it is not recommendable to use control of the pump power as a means to control the delay advancement.

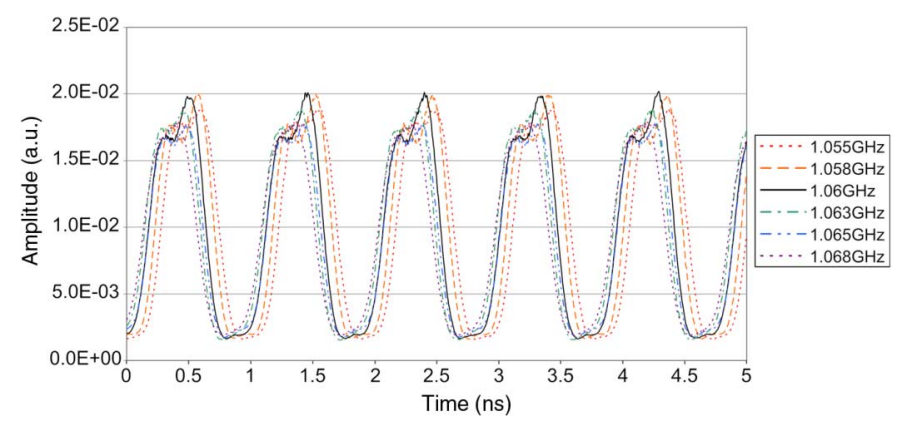

Fig. 12. Delay/advancement and simultaneous amplification of a square signal.

One important characteristic of the graph in Fig. 11 is that all the curves intersect at one point around $1.06 \mathrm{GHz}$. This is the point of equal spacing of Brillouin gain lines and probe harmonics. Therefore, at this point, there should be no induced delay regardless of the pump power (i.e., the curves should cross and do this at $0 \mathrm{~ns}$ ). The reason why this intersection point is not exactly at 0-ns delay is because of an extra delay offset introduced by signal distortion (in passing from the square to the quasisinusoidal waveform, the peak of the sinusoid does not coincide exactly with the center of the flat top of the square signal).

The retiming results presented so far introduce strong signal reshaping. However, as demonstrated in Fig. 12, this technique can also be used to simultaneously amplify and retime a periodic signal with relatively little distortion. This result was obtained for 27-dB Brillouin gain and a seven-gain line Brillouin comb (again note that the first two lines were filtered by the FBG filter that rejects the pump). The total retiming range of this signal is $\sim 0.12 \mathrm{~ns}$. This reduction in the total delay/advancement range comes as a result of the increment in the number of lines in the comb [as indicated by (3)]. As commented upon previously, the requirement for low distortion (high number of gain lines) is opposite that of the high retiming range, so a compromise must be struck.

We have observed that when slightly detuning the probe from the Brillouin frequency shift, the output signal presents lower distortion levels. In fact, the results of Fig. 12 were obtained by downshifting the probe by $9.29 \mathrm{GHz}$ (instead of the $9.3 \mathrm{GHz}$ of the Brillouin frequency shift). Even though the exact reasons for this are not understood presently, it seems that with this shift there is a more homogeneous gain distribution between the different harmonics.

\section{CONCLUSION}

This paper presents a versatile all-optical signal-processing technique for periodic signals using a Brillouin gain comb. As has been discussed, this technique provides control over the amplitude and phase of the input signal. This way, the signal can easily be retimed and/or reshaped. An important characteristic of this technique is that it is frequency independent (i.e., there is no pump power penalty as the repetition rate of the input signal is increased).

Furthermore, the technique offers some unique features that cannot be replicated with conventional Brillouin-assisted slow light. Thus, for example, the signals can be delayed with very 
small gain variation, amplified without inducing an extra delay, and high-gain fast light can be generated.

\section{ACKNOWLEDGMENT}

The authors would like to thank Furukawa Electric Co. (Japan) and Dr. K. Mukasa for the loan of the highly nonlinear fiber used in the experiment.

\section{REFERENCES}

[1] T. Horiguchi, K. Shimizu, T. Kurashima, M. Tateda, and Y. Koyamada, "Development of a distributed sensing technique using Brillouin scattering," J. Lightwave Technol., vol. 13, no. 7, pp. 1296-1302, Jul. 1995.

[2] A. Loayssa, D. Benito, and M. J. Garde, "Optical carrier Brillouin processing of microwave photonic signals," Opt. Lett, vol. 25, pp. 1234-1236, Sep. 2000.

[3] X. S. Yao, "Phase-to-amplitude modulation conversion using Brillouin selective sideband amplification," IEEE Photon. Technol. Lett., vol. 10, no. 2, pp. 264-266, Feb. 1998.

[4] M. G. Herráez, K. Y. Song, and L. Thévenaz, "Optically controlled slow and fast light in optical fibers using stimulated Brillouin scattering," Appl. Phys. Lett., vol. 87, pp. 081113-1-081113-3, Aug. 2005.

[5] Y. Okawachi, M. S. Bigelow, J. E. Sharping, Z. Zhu, A. Schweinsberg, D. J. Gauthier, R. W. Boyd, and A. L. Gaeta, "Tunable all-optical delays via Brillouin slow light in an optical fiber," Phys. Rev. Lett., vol. 94, pp. 153902-1-153902-4, Apr. 2005.

[6] C. Jáuregui, P. Petropoulos, and D. J. Richardson, "Slowing of pulses to c/10 with sub-watt power levels and low latency using Brillouin amplification in a bismuth oxide optical fiber," J. Lightwave Technol., vol. 25, no. 1, pp. 216-221, Jan. 2007.

[7] K. Y. Song, K. S. Abedin, K. Hotate, M. G. Herráez, and L. Thévenaz, "Highly efficient Brillouin slow and fast light using $\mathrm{As}_{2} \mathrm{Se}_{3}$ chalcogenide fiber," Opt. Expr. vol. 14, pp. 5860-586, Jun. 2006. [Online]. Available: http://www.opticsinfobase.org/abstract.cfm?URI=oe-14-13-5860.

[8] M. G. Herráez, K. Y. Song, and L. Thévenaz, "Arbitrary-bandwidth Brillouin slow light in optical fibers," Opt. Expr. vol. 14, pp. 1395-1400, Feb. 2006. [Online]. Available: http://www.opticsinfobase.org/abstract.cfm?URI=oe-14-4-1395.

[9] Z. Zhu, A. M. C. Dawes, D. J. Gauthier, L. Zhang, and A. E. Willner, "Broadband SBS slow light in an optical fiber," J. Lightwave Technol., vol. 25, no. 1, pp. 201-206, Jan. 2007.

[10] L. Yi, L. Zhan, W. Hu, and Y. Xia, "Delay of broadband signals using slow light in stimulated Brillouin scattering with phase-modulated pump," IEEE Photon. Technol. Lett, vol. 19, no. 8, pp. 619-621, Apr. 2007.

[11] E. Lichtman, R. G. Waarts, and A. A. Friesem, "Stimulated Brillouin scattering excited by a modulated pump wave in single-mode fibers," J. Lightwave Technol., vol. 7, no. 1, pp. 171-174, Jan. 1989.

[12] S. Chin, M. Gonzalez-Herraez, and L. Thévenaz, "Zero-gain slow \& fast light propagation in an optical fiber," Opt. Expr. vol. 14, pp. 10684-10692, Oct. 2006. [Online]. Available: http://www.opticsinfobase.org/abstract.cfm?URI=oe-14-22-10684.

[13] T. Schneider, R. Henker, K. U. Lauterbach, and M. Junker, "Comparison of delay enhancement mechanisms for SBS-based slow light systems," Opt. Expr. vol. 15, pp. 9606-9613, Jul. 2007. [Online]. Available: http://www.opticsinfobase.org/abstract.cfm?URI=oe-15-15-9606.

[14] M. Fujiwara, J. Kani, H. Suzuki, K. Araya, and M. Teshima, "Flattened optical multicarrier generation of $12.5 \mathrm{GHz}$ spaced 256 channels based on sinusoidal amplitude and phase hybrid modulation," Electron. Lett., vol. 37, pp. 967-968, Jul. 2001.

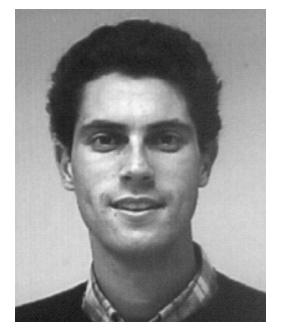

César Jáuregui Misas was born in Santander, Spain, in 1975. He received the Telecommunication Technical Engineering and Telecommunication Engineering degrees from the University of Cantabria, Cantabria, Spain, in 1996 and 1998, respectively, where he received the Ph.D. degree in 2003.

In 2004, he was with the Public University of Navarra and the University of the Basque Country. After visiting the Optoelectronics Research Centre at Southampton University, Southampton, U.K., to carry out a postdoctoral stay funded by the Fundación Ramón Areces, he moved to the Institute of Applied Physics at the University Friedrich-Schiller, Jena, Germany. His primary research concern is the interrogation of fiber-optic sensors, slow-light, and nonlinear effects in optical fibers and fiber lasers. He has coauthored more than 90 papers presented in conferences and scientific journals.

Dr. Misas has been awarded with several academic prizes. In 2004, he was awarded a prize for the best thesis at the University of Cantabria

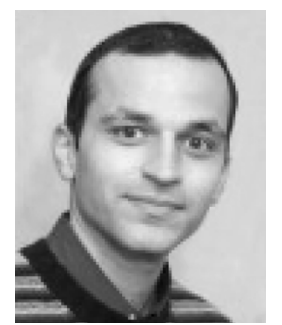

Periklis Petropoulos was born in Patras, Greece. He received the M.Sc. degree in communications engineering from the University of Manchester Institute of Science and Technology, Manchester, U.K., in 1996 and the Ph.D. degree in optical telecommunications from the Optoelectronics Research Centre (ORC), University of Southampton, U.K., in 2000.

Currently, he is Southampton Reader at the ORC. His particular areas of interest and expertise lie in the fields of optical communications and nonlinear fiber technology. Within the Optical Fibre Communications Group at the ORC, he is working on areas, such as all-optical processing and switching in optical fibers; pulse manipulation for optical communications using fiber Bragg gratings, including applications in optical correlation systems for the implementation of optical code-division multiple access and optical packet-switched systems; silica and compound glass holey fibers and their nonlinear applications; and fiber lasers. His research has produced more than 180 papers in journals and conferences as well as four international patents in the field of optical physics and optical communications.

Dr. Petropoulos is a member of the Optical Society of America.

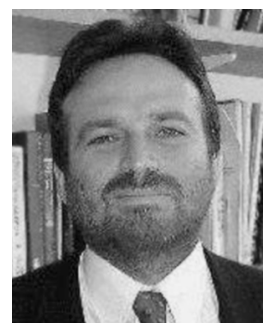

David J. Richardson received the B.Sc. and Ph.D. degrees in fundamental physics from Sussex University, Brighton, U.K., in 1985 and 1989, respectively.

He joined the Optoelectronics Research Centre (ORC) at Southampton University, Southampton, U.K., as a Research Fellow in 1989. Currently, he is Deputy Director of the ORC, where he is responsible for optical-fiber device and systems research. His current research interests include microstructured fibers, high-power fiber lasers, short-pulse lasers, optical-fiber communications, and nonlinear fiber optics. He has published more than 600 conference and journal papers in his 19 years at ORC and has produced over 20 patents. He is a frequent invited speaker at the leading international optics conferences in the optical communications, laser, and nonlinear optics fields and is an active member of the national and international optics communities.

Prof. Richardson is a Fellow of the Optical Society of America. He was awarded a Royal Society University Research Fellowship in 1991 in recognition of his pioneering work on short-pulse fiber lasers. 